\title{
3D nanoprinted high NA ultra-compact catadioptric lens for efficient collection in forward detection configuration microscopy
}

\author{
Andrea Bertoncini ${ }^{1}$, Siarhei P. Laptenok ${ }^{1}$, Vijayakumar P. Rajamanickam ${ }^{1}$, Carlo Liberale ${ }^{1}$ \\ 1. King Abdullah Univiversity of Science and Technology, Thuwal, Saudi Arabia
}

In optical microscopies which implement a forward detection scheme such as Coherent Anti-stokes Raman Scattering (CARS), Stimulated Raman Scattering (SRS) or Second - and Third- Harmonic Generation, efficient detection is usually achieved by collecting the signal with high Numerical Aperture (NA) objectives. While the use of high-end well-corrected high-NA microscope objectives for excitation is necessary to achieve high resolution, to efficiently induce non-linear effects and to have excellent imaging capabilities, the detection optics only requires the collimation of the highly divergent beam coming from the excitation path. Moreover, high NA objectives feature short working distances which render them incompatible with top stage incubators, which are the solution of choice for controlled long term experiments on live cells. Among the mentioned techniques, SRS strictly requires the collection of the full excitation beam NA to avoid spurious background signal caused by cross phase modulation (XPM) which can spoil the sensitivity of the system [1].

Here we show the design and fabrication of a high-NA 3D nanoprinted ultra-compact catadioptric lens that can replace a high NA commercial microscope objective, and show its use for the full forward collection of Stimulated Raman scattering microscopy signal. The lens is composed by multiple refractive and reflective elements, which have dimensions smaller than $100 \mu \mathrm{m}$, and has been fabricated on a $170 \mu \mathrm{m}$ thick coverslip glass substrate with a commercial two-photon lithography-based system (Nanoscribe GmbH) using the proprietary resist IP-S (Nanoscribe). The lens has a NA of 1.2 in water immersion, with a working distance of $1.35 \mathrm{~mm}$, a diameter of 6 $\mathrm{mm}$ and a total thickness of $170 \mu \mathrm{m}$ (coverslip) $+70 \mu \mathrm{m}$ (lens). The working principle of the lens is illustrated in Fig 1a.

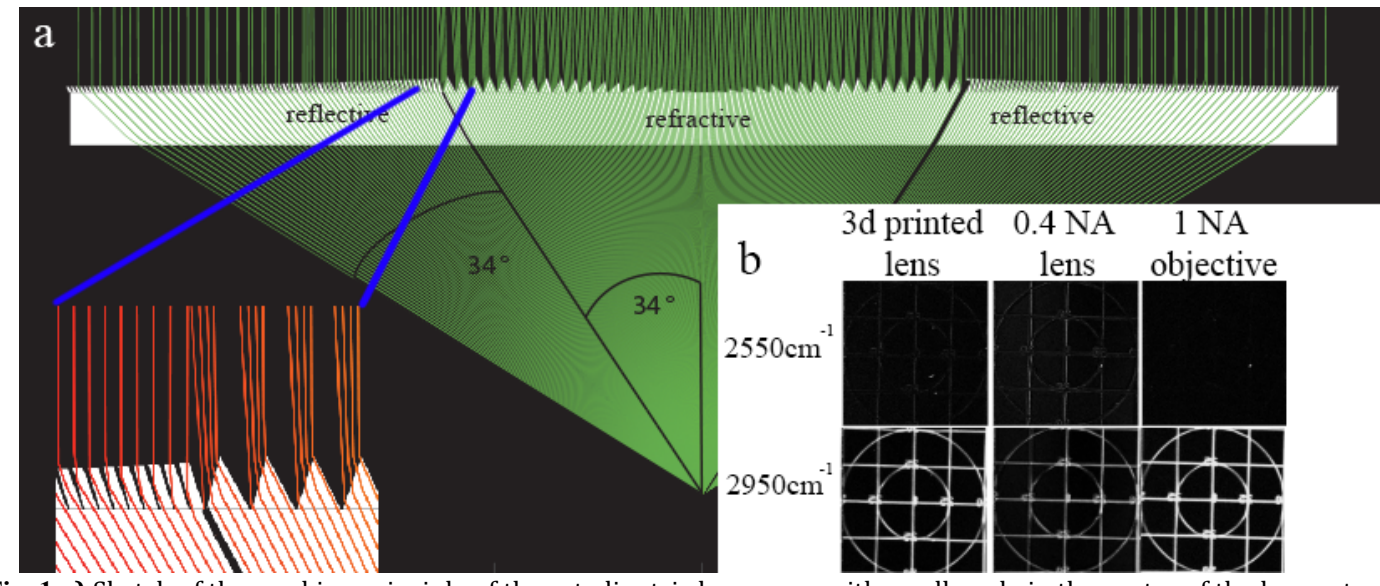

Fig. 1 a) Sketch of the working principle of the catadioptric lens : rays with small angle in the center of the lens gets collimated by refraction, while rays with bigger angles in the periphery of the lens gets collimated by total internal reflection. b) SRS imaging of a 3D printed grid made of IP-S by using different collection optics at non-resonant $\left(2550 \mathrm{~cm}^{-1}\right)$ and resonant $\left(2950 \mathrm{~cm}^{-1}\right)$ Raman shift

To demonstrate the imaging capabilities of our lens, we printed a regular grid of IP-S on a coverslip and then we performed SRS imaging by using different collection optics (fig. 1b), namely a $3 \mathrm{~d}$ printed lens, a f=30mm 0.4 NA diameter planoconvex lens and a 40x 1 NA high-end water immersion microscope objective. The excitation beams are focused on the sample by a 60x 1.2 NA water immersion objective. The obtained SRS images, collected at a resonant wavenumber for the polymer material $\left(2900 \mathrm{~cm}^{-1}\right)$, show a comparable signal for the $3 \mathrm{~d}$ printed lens and for the 40x microscope objective. The SRS images collected at a non-resonant wavenumber $\left(2550 \mathrm{~cm}^{-1}\right)$ show the XPM induced background signal coming from the polymer grid in the case of collection with the 0.4 NA lens while in the case of the $3 \mathrm{~d}$ printed lens and of the $40 \mathrm{x}$ microscope objective the signal is negligible. This means that the full NA is collected from the $3 \mathrm{~d}$ printed lens and the XPM background is avoided.

Our lens can be printed in few hours and replace bulky and expensive high NA microscopy objectives in forward detection configurations, with the further advantage that it can fit in narrow environments such as table top incubators. This 3D printed high-NA collimating optics will be used to perform long term in-vitro SRS experiments on live cells with the complete control on the environmental parameters. 